\title{
MECHANISTIC AND SYNTHETIC ASPECTS OF ORGANOMETALLIC OXIDATIVE ADDITIONS
}

\author{
John J. Eisch
}

Department of Chemistry, The State University of New York at Binghamton, Binghamton, New York 13901, USA

\begin{abstract}
The electron transfer occurring between subvalent metals and organic compounds often plays a pivotal role in the syntheses and the reaction mechanisms of organometallic compounds. This presentation examines the factors that promote such electron transfer, namely the metal's oxidation potential, the organic substrate's electron affinity, the autoassociation of $R_{n} M$ units and the coordination on the metal of ligands or donor solvent. Examples of such electron-transfer processes with maingroup and transition metal complexes are offered for: a) the formation of carbon-carbon and carbon-element bonds; b) the cleavage of carbon-heteroatom bonds; c) the oligomerization of unsaturated hydrocarbons; and d) the action of $\mathrm{RMgX}$ and $\mathrm{RLi}$ on olefins or acetylenes bearing electronegative substituents .
\end{abstract}

\section{INTRODUCTION}

The interaction of zerovalent metals with various organic substrates, such as unsaturated hydrocarbons, halides, ethers or even other organometallics constitutes a versatile method for the preparation of main-group and transition-metal organometallic compounds $(1,2)$. In a formal sense, such reactions may be viewed as oxidative additions, whereby the metal transfers electron density to the organic substrate and thereby gains in oxidation number (eq. 1):

$$
M+R-E \stackrel{\mathrm{k}_{1}}{\longrightarrow} \mathrm{R}-\mathrm{M}-\mathrm{E} \text { or } \mathrm{R}-\mathrm{E} \cdot \mathrm{M}
$$

In organometallic synthesis (3) and in the study of reaction mechanisms (4) it is of cardinal importance to understand the factors determining the rate $\left(k_{1}\right)$ and the equilibrium constant $\left(\mathrm{K}_{1}\right)$ of equation 1 .

This view of metal reactions as electron-transfer processes can be carried over to the reactions of organometallics themselves, in which view both the rate $\left(k_{2}\right)$ and the equilibrium constant $\left(\mathrm{K}_{2}\right)$ are ascribed to the ease of electron transfer from the carbon-metal bond (eq. 2):

$$
\mathrm{R}^{\prime}-\mathrm{M}+\mathrm{R}-\mathrm{E} \stackrel{\mathrm{k}_{2}}{\longrightarrow} \mathrm{R}^{\prime} \cdot \mathrm{M}^{+}+(\mathrm{R}-\mathrm{E})^{-} \longrightarrow \text { Products }
$$

The suggestion that organometallic reactions might proceed via such single-electron transfer (SET) is at least 50 years old, for it was invoked by Blicke and Powers to explain the reduction products encountered in the reactions of ketones with Grignard reagents (5). In recent years, Ashby has adduced impressive experimental evidence for the occurrence of just such SET processes with ortho-substituted benzophenones and branched alkylmagnesium halides (6). Furthermore, by application of appropriate chemical trapping or instrumental detection, freeradical transients now have been shown to be intermediates in a large number of organometallic processes.

This presentation wishes to explore the utility of treating the reactions of both metals and organometallic compounds as electron-transfer processes. Offered for consideration are selected studies carried out in the author's laboratory that involve alkali metals, magnesium, nickel, cobalt and titanium. In defense of drawing this parallel in behavior between metals and organometallics, the author refers with approval to the fruitful suggestion of Rundle that metallic character arises from metals having insufficient valence electrons to fill the available, low-energy bonding orbitals. The resulting "electron-deficient" systems would therefore display greater delocalization. Rundle recognized that metals represented only an extreme stage in such delocalization and that alloys (M-M'), interstitial compounds (M-E), 
organometallic compounds $(M-R)$ and organic compounds (R-R) were systems of successively greater electron localization (lesser electron deficiency) (7) (eq. 3).

$$
\mathrm{M}_{\mathrm{n}}>\left(\mathrm{M}-\mathrm{M}^{\prime}\right)_{\mathrm{n}}>(\mathrm{M}-\mathrm{E})_{\mathrm{n}}>(\mathrm{R}-\mathrm{M})_{\mathrm{n}}>\mathrm{R}-\mathrm{M}>\mathrm{R}-\mathrm{R}
$$

As corollaries of this view, one can expect that the "metallic character" of an organometallic should be greater: a) in associated or clustered systems, $(\mathrm{RM}) \mathrm{n}$, than in monomeric $\mathrm{R}-\mathrm{M}$; b) in monomeric $\mathrm{R}-\mathrm{M}$, as $\mathrm{M}$ is more electropositive (cf, eq. 2) and c) in monomeric $\mathrm{R}-\mathrm{M}$, where $R$ forms a relatively stable radical (cf. eq. 2). Illustrative of the last two points is the observation that t-butyllithium reacts with diphenylacetylene by electron-transfer, while n-butyllithium and triethylaluminum react by simple addition to the triple bond $(8-10)$.

\section{ALKALI METAL ADDITION TO UNSATURATED SYSTEMS}

Genera1 Considerations

The thermodynamic factors operative in the reaction of alkali metals with unsaturated organic compounds can be appreciated by means of a Born-Haber cycle (2). With appropriate modifications, the free energy of solution or solution potential (P) can be expressed in terms of the free energies required to convert bulk metal and organic substrate into solvated, associated organometallic $(\mathrm{RM})_{\mathrm{n}}$, where $\mathrm{M}$ is either a main-group or a transition metal (eq. 4). Finally, estimates of the free energies for sublimation (S), ionization (I), electron affinity $(E)$, solvation $(H)$ and association (A) can be made from known enthalpy values $(\Delta H)$, with the understanding that suitable entropy contributions $(\Delta \mathrm{S})$ would be involved (Fig. 1$)$ :

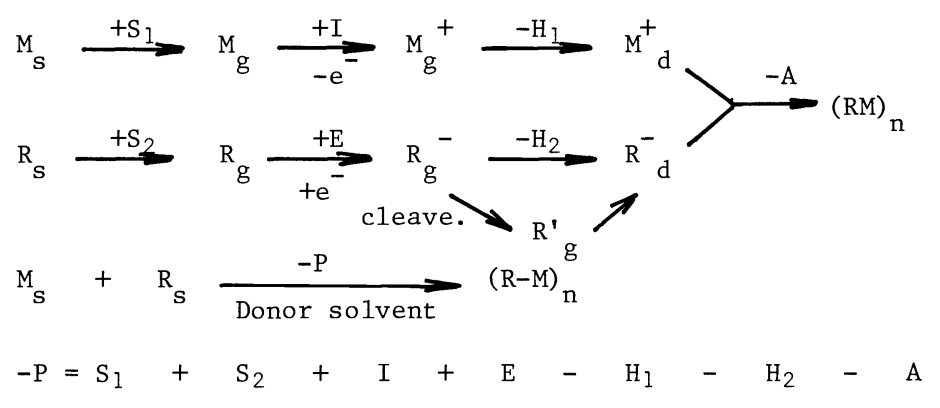

Fig. 1 Born-Haber cycle for forming $(\mathrm{RM})_{\mathrm{n}}$

From Fig. 1 it is readily seen that solvation or coordination of the metal center by donor ligands $\left(\mathrm{H}_{1}\right)$ and the association or bonding between $M$ and $R(A)$ are decisive in lowering the free energy of the system $(P)$. Conversely, the principal energy investments are those involved in: a) disrupting the metal lattice $\left(\mathrm{S}_{1}\right)$; b) transferring electrons from metal to organic substrate $(I+E)$; and $c)$ in some cases, cleaving a bond in $R(C-C, C-X, C-O$, etc.) to form $R^{\prime}$.

This Born-Haber treatment makes comprehensible many thermodynamic aspects of metal reactions, ranging from the high solution potentials of cesium (small $\mathrm{S}_{1}$ and I) and of lithium (large $\mathrm{H}_{1}$ to compensate for large $\mathrm{S}_{1}$ and $\mathrm{I}$ ) to the success of metal vapor synthesis (prior investment of $S_{1}$ to form $M_{g}$ as reactant). Of equal importance, however, is the observed validity of such Born-Haber considerations for the kinetic course of many metal reactions. The rates with which metals react with organic substrates often increase as the metal has a lower ionization potential $\left(\mathrm{S}_{1}, \mathrm{Cs}>\mathrm{K}>\mathrm{Li}\right.$ ) or a larger solvation as cation $\left(\mathrm{H}_{1}, \mathrm{Li}>\mathrm{Na}>\mathrm{Mg}\right)$. In a parallel fashion, the greater the electron affinity (smaller $+\mathrm{E})$ and the solvation $\left(\mathrm{H}_{2}\right)$ of the organic substrate, often the more facile is the reaction (especially when $R$ must undergo cleavage to $\mathrm{R}^{\prime}$, as in $\mathrm{R}=$ ether, halide, amine, etc.). Such a correlation of kinetics with equilibrium factors does not follow a priori from thermodynamics but must be established a posteriori from experiment. Such metal-organic substrate interactions may be another illustration of the Hammond thermic postulate (11). Such an extrathermodyanamic hypothesis suggests that highly exothermic reactions (in Fig. 1, dominance of $H_{1}$ and A) would have small activation energies and therefore high rates. Implied in the small activation energy is a small change in geometry in going from the starting reagents to the transition state. One would accordingly expect that ground-state (thermodynamic) factors would be pertinent in lowering the activation energy. Where application of this postulate is valid, the rapidity of electron transfer, as in eq. 5 for SET or eq. 1 for the general case, should reflect the same factors considered in the Born-Haber treatment (Fig. 1): 


$$
\mathrm{M}_{\mathrm{S}}+\mathrm{R}_{\mathrm{S}} \longrightarrow\left(\mathrm{k} \cdot \mathrm{M}_{\mathrm{d}} \longrightarrow\right. \text { Products }
$$

Furthermore, in keeping with the above-drawn parallel between the behavior of metals and organometallics, one might expect that solvation, ionization and electron affinity factors would also be significant in organometallic reactions. Accordingly, a Born-Haber cycle that incorporates the Blicke-Powers hypothesis can be constructed, as in Fig. 2:

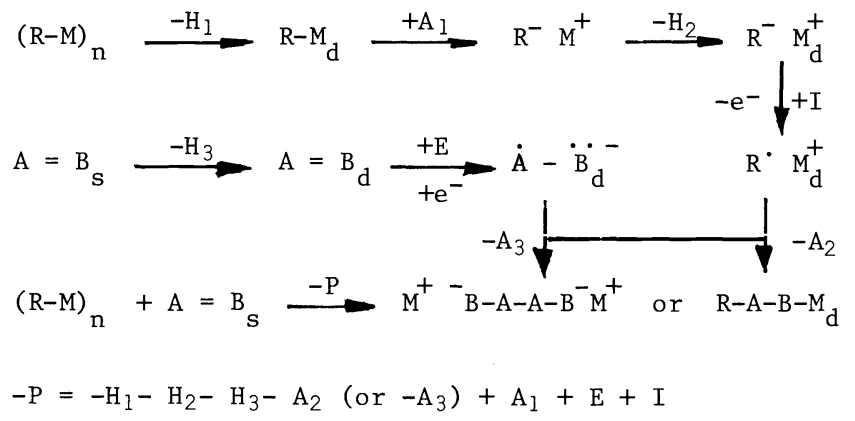

Fig. 2 Born-Haber cycle for adding $(\mathrm{RM})_{n}$ to organic substrate $A=B$.

In agreement with the postulated intermediacy of free radicals, one observes both normal adducts (R-A-B-M) and reductive coupling products. Thus, benzophenone and tritylsodium yield the salt of benzopinacol and the dimer of the triphenylmethyl radical (12) (eq. 7):

$$
2 \mathrm{Ph}_{3} \mathrm{CNa}+\mathrm{Ph}_{2} \mathrm{CO} \longrightarrow \underset{\mathrm{NaO}}{\left(\mathrm{Ph}_{3} \mathrm{C}\right)_{2}+\underset{\mathrm{ONa}}{\mathrm{Ph}_{2}} \mathrm{C}-\mathrm{CPh}_{2}}
$$

With reference to the reaction potential $\mathrm{P}$, one would expect that such electron transfer would be favored, both thermodynamically and kinetically, by strong-donor solvents $\left(\mathrm{H}_{1}\right.$ and $\left.\mathrm{H}_{2}\right)$, by easily ionized $\mathrm{R}$ anions (I) and by electron-attracting substrates (sma11 $+\mathrm{E}$ ). All these expectations are realized in the laboratory: eq. 7 shows the validity of a small I for the trityl anion; the occurrence of reductive coupling is frequent with carbonyl compounds (eq. 7), showing the fostering effect of a small +E; and strong donor solvents, such as hexamethylphosphorus triamide, are known to favor electron-transfer processes with Grignard reagents ((13), large $\mathrm{H}_{1}$ and $\left.\mathrm{H}_{2}\right)$.

Illustrative reactions of alkali metals with substrates.

A telling illustration of the effects of solvent and metal on electron-transfer processes is seen in the reductive dimerization of benzalaniline depicted in Fig. 3. In solvents of low donor character $\left(\mathrm{C}_{6} \mathrm{H}_{6}\right.$ or $\left.\mathrm{Et}_{2} \mathrm{O}\right)$, a variety of metals ( $\left.\mathrm{Na}, \mathrm{Mg}, \mathrm{Al}\right)$ slowly converts the anil into approximately an equimolar mixture of meso and $\mathrm{d}, 1$ dimers. However, strong donor solvents (THF) and active metals ( $\mathrm{Na}, \mathrm{Li}$ ) rapidly dimerize the anil to preponderantly the dl dimer $(>90 \%)$. This stereoselectivity can be rationalized in terms of the preferred pre-association of radical anions (14).

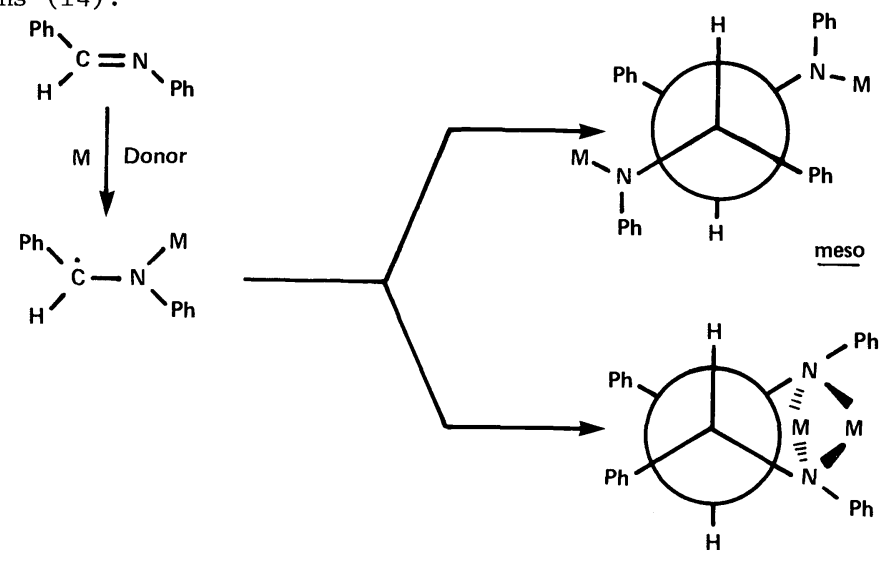

\footnotetext{
Fig. 3 The alkali-metal coupling of benzalaniline
}

In order to circumvent the energy necessary to disrupt the metal lattice (S), one can resort to metal vapor or employ soluble metal-substrate adducts for more rapid metal reactions. The iynthetic utility of the dilithium adduct of biphenyl in smoothly cleaving $\mathrm{C}-\mathrm{C}, \mathrm{C}-\mathrm{X}, \mathrm{C}-\mathrm{N}$ and 
C-O bonds is portrayed in Fig. 4 (15).

$\mathrm{ArLi}+\mathrm{Ar}_{2} \mathrm{NLi}$

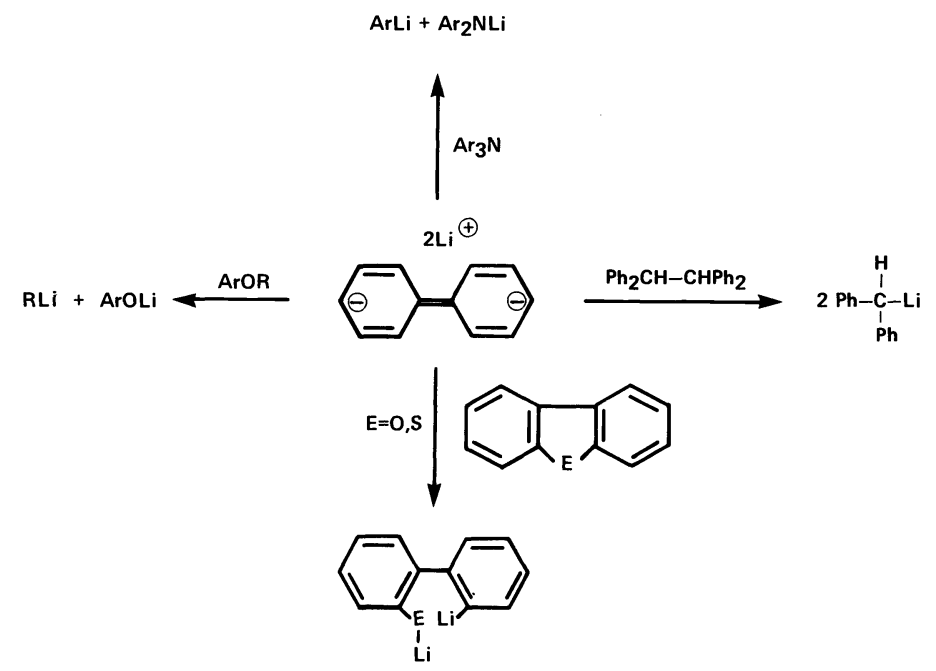

Fig. 4 Carbon-element bond cleavages with dilithium biphenylide

The practicality of the procedure pivots on the biphenyl adduct permitting electron transfer in a homogeneous medium and on its electron affinity $\left(E_{B}\right)$ being comparable or less than that of the reactants $\left(E_{R}\right.$, i.e.: $\left.+E_{B}>+E_{R}\right)$.

Substituents on the substrate $A=B$ can profoundly alter the reaction potential ( $P$ ) for metal reactions by increasing the substrate's electron affinity (smaller + E). Particularly striking is the influence of an organosilyl group on olefinic, acetylenic and aromatic systems. Thus, although simple olefins show little tendency to form alkali metal adducts, vinylsilanes do so readily and can be reductively dimerized in high yield (>80\%, Fig. 5, (16)):

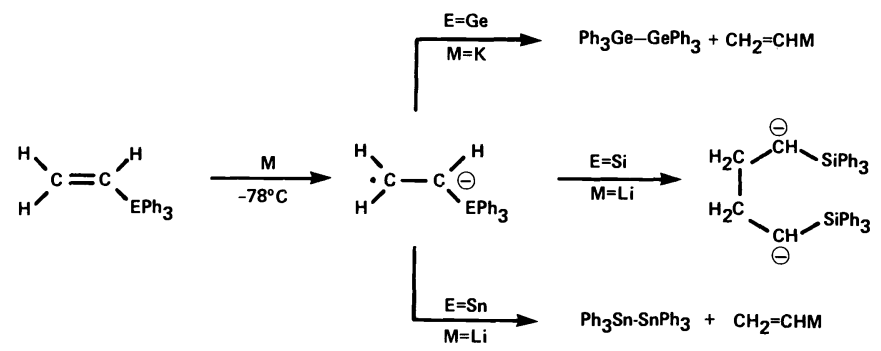

Fig. 5 Alkali-metal coupling of Group IV vinyl derivatives

It is noteworthy that not only can the dimerization of the vinylsilane be readily rationalized in terms of radical-anion intermediates, but also the dimerizing cleavage of the analogous germanium and tin compounds as well (17).

Similarly, arylsilanes show an enhanced tendency to form metal adducts when compared with alkylbenzenes. The reaction illustrated in Fig. 6 also emphasizes the importance of temperature in intercepting transient radical-anions. At $-78^{\circ} \mathrm{C}$ with $\mathrm{M}=\mathrm{K}$ or $\mathrm{Li}$ and with $\mathrm{THF}$ or $\mathrm{DME}$, greater than $80 \%$ yields of the para-coupled dimer can be formed (18). In this case, the presence of the radical-anion can be corroborated by ESR spectroscopy (19). At room temperature, only $\mathrm{R}_{3} \mathrm{SiM}$ products are produced.
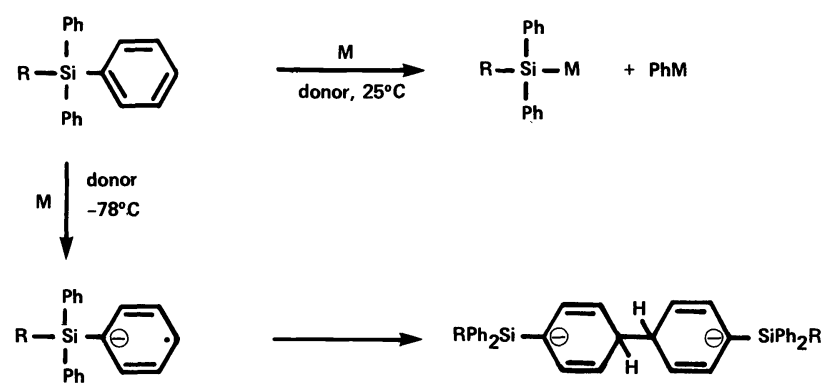

Fig. 6 Para-coupling of phenylsilanes by alkali metals 
Finally, the course taken by various alkali metal-induced cleavage reactions can be adduced as unambiguous evidence for the determining role of radical-anion intermediates. As outlined in Fig. 7, the cleavage of alkyl aryl sulfones by sodium amalgam and alcohols can be ascribed to the formation of a radical-anion (presumably in the aryl group) and its fragmentation to the arylsulfenate anion and an alkyl radical. Both the sulfenate anion and the disproportionation products of the radical have been observed (20).

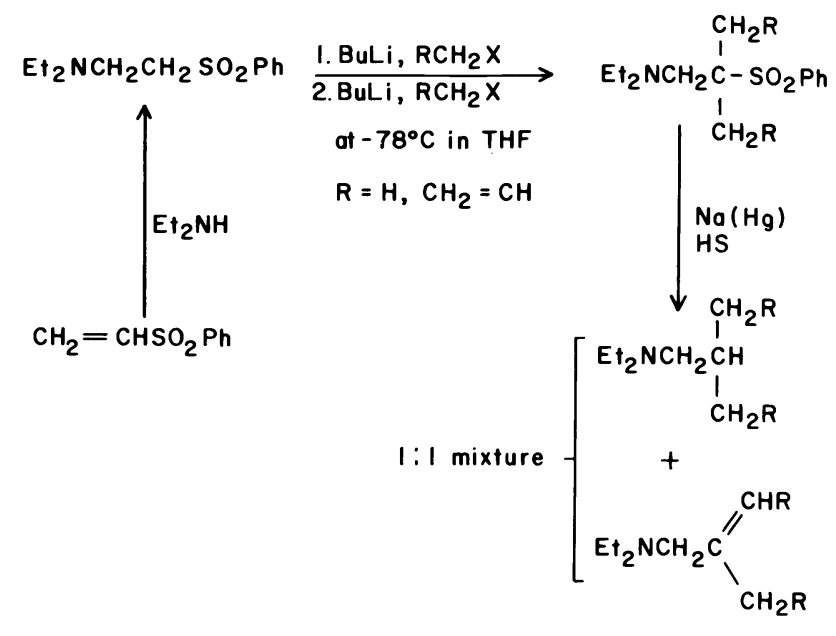

Fig. 7 Alkali-metal cleavage of sulfones via radical-anions Electron-transfer reactions of organolithium and organomagnesium reagents

Countless recent studies could be cited to support the widespread occurrence of SET processes with organometallics. For illustrative purposes, however, two unusual reactions encountered in this laboratory will be offered. Since both involve $\alpha, \beta$-unsaturated sulfones, these reactions may again show how the electron affinity of an olefin or acetylene is enhanced by electron-withdrawing substituent (cf. reactions of silanes above and Note a.). In one reaction, originally observed by Truce (21), acetylenic sulfones yield disubstituted acetylenes ( $R-C \equiv C-R^{\prime}$ ) with RLi or RMgX. We have shown that this substitution does not occur by any addition-elimination pathway (Fig. 8), but rather by an SET process (Fig. 9).

\section{TRUCE REACTION}

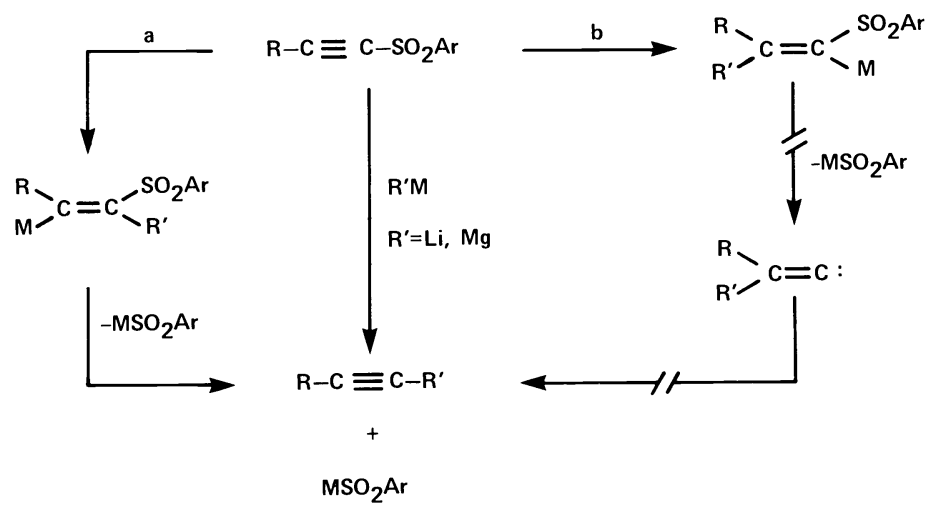

Fig. 8 The Truce reaction and its unlikely addition pathways

Diagnostic for the occurrence of an SET pathway were products derived from the acetylenic sulfone and 5-hexenylmagnesium chloride, namely a $2: 1$ mixture of the open-chain and cyclic acetylenes (22). The formation of cyclopentylmethyl phenyl sulfone means that the 5-hexenyl radical is formed and has an opportunity to cyclize before coupling to form the product (Fig. 9):

Note $\mathrm{a}$ : Both the $\mathrm{RSO}_{2}$ and $\mathrm{R}_{3} \mathrm{Si}$ may be considered electron-withdrawing, but for different reasons: $\mathrm{RSO}_{2}$ withdraws electrons both by delocalization and induction (-T, $-\mathrm{I}$ ), while $\mathrm{R}_{3} \mathrm{Si}$ withdraws electrons only by delocalization $(-\mathrm{T},+\mathrm{I})$. 


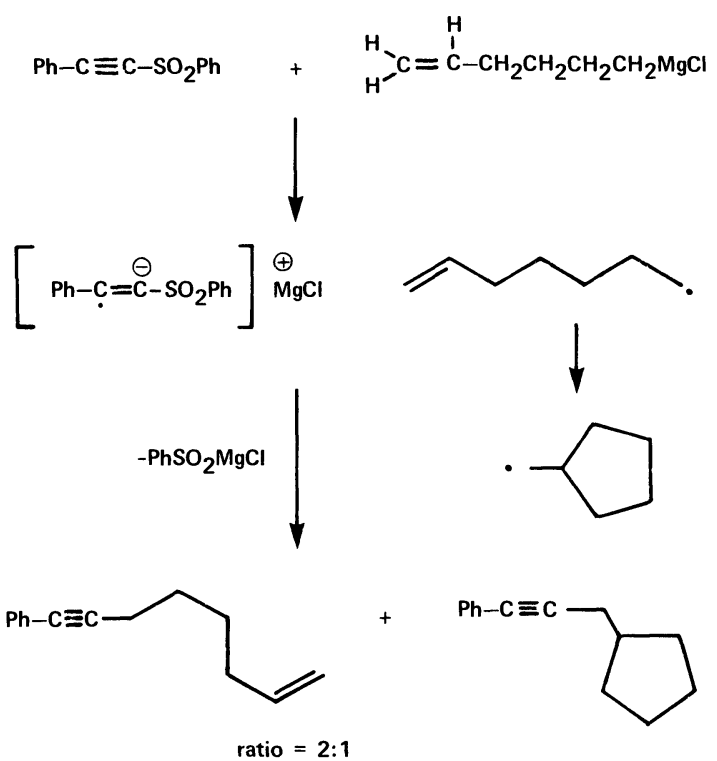

Fig. 9 SET-reaction products from phenyl phenylethynyl sulfone

A second example is the alkylating cyclization observed when phenyl trans-3-bromopropen-2-yl sulfone is treated with certain Grignard reagents (23) (Fig. 10). Especially noteworthy is that the dimethylallyl group is attached to the cyclopropyl ring by the more hindered terminus of the allyl group. Such preference is inexplicable on steric grounds, but would be readily reconcilable in SET terms. The spin density of the intermediate dimethylallyl radical should be greater at the tertiary carbon terminus.

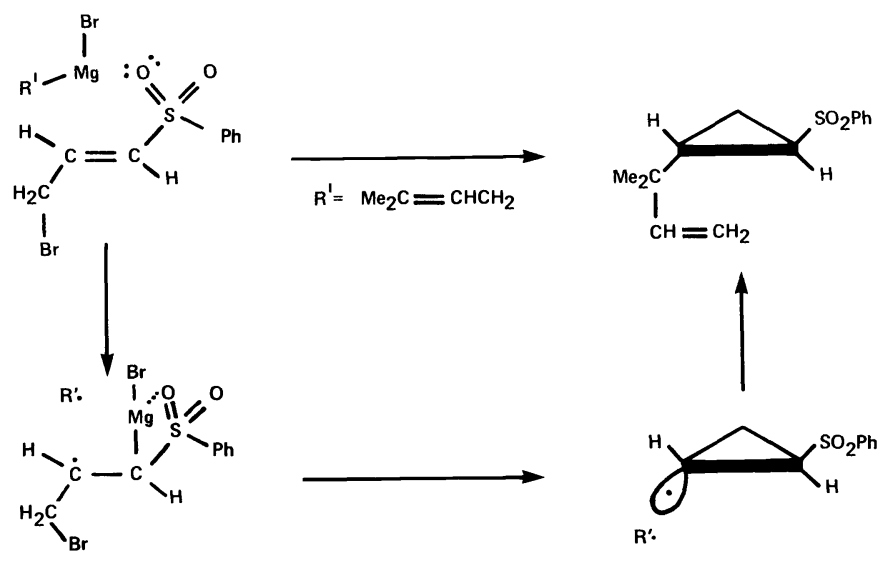

N.B. : The cis - 3 - bromopropenyl sulfone does not yield any cyclopropyl sulfone.

Fig. 10 Allylative cyclization of 3-propenyl sulfones

\section{TRANSITION-METAL ELECTRON-TRANSFER REACTIONS}

Thermodynamic and kinetic factors

With reference to the Born-Haber cycle in Fig. 1, it should be noted that transition-metal organometallics $(\mathrm{RM})_{n}$, may formally be zerovalent metal complexes but still involve varying degrees of charge transfer from the metal to the ligand ( $d_{\pi} \rightarrow p_{T}$ retrodative bonding). Furthermore, either mononuclear $(n=1)$ or metal cluster $(n>2){ }^{\pi}$ complexes could be formed. Finally, although bulk solvent effects are also important in determining $\mathrm{H}_{l}$, specific solvation or ligand coordination ( $L$ ) to the metal will make the larger contribution to $\mathrm{H}_{1}$. Because transition metal centers usually display a maximum coordination number for both electronic and steric reasons, such specific, firm solvation can interfere with the reaction of $R M-L_{n}$ and organic substrates. Evidence indicates that a given substrate often must coordinate with the metal center for subsequent reaction. Thus, although a maximum number of ligands may favor the formation of $(\mathrm{RM})_{\mathrm{n}}$, because of a favorable $\mathrm{H}_{1}$, such coordination lowers the reactivity of the complex. 
Deoxygenation of epoxides

Illustrative of the importance of donor ligands and of an available coordination site on the metal is the deoxygenation of epoxides by nickel(0) complexes. As shown in Fig. 11, bis1,5-cyclooctadiene-nickel(0) does not react with epoxides, whereas addition of one equivalent of bipyridyl gives an active agent, namely bipyridy1(1,5-cyclooctadiene)nickel. But an excess of bipyridyl destroys the reactivity, since the nickel thereby forms the bisbipyridylnicke1, which is coordinatively saturated (24):

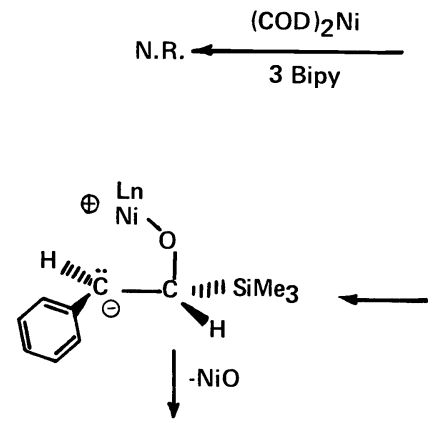

trans-olefin
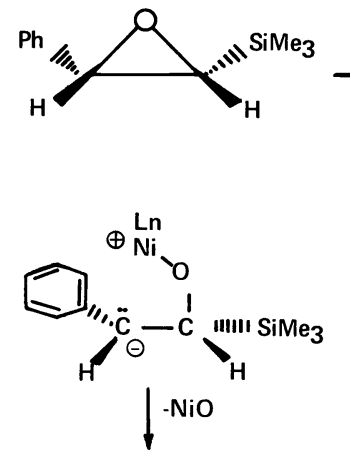

cis-olefin

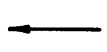

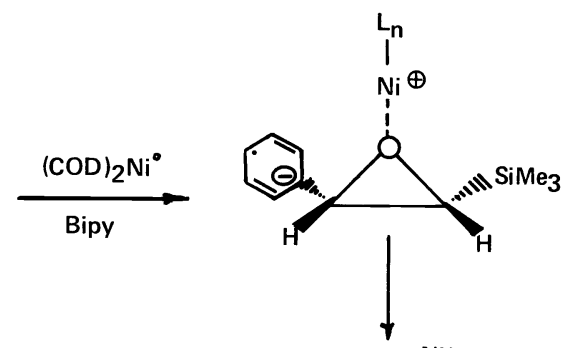

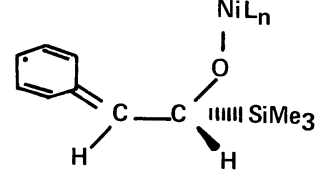

Fig. 11 Deoxygenation of epoxides by nicke1(0) complexes

The SET pathway shown is favored because of the lack of stereoselectivity in the olefin formed and because of the failure of alkyl-substituted epoxides to undergo deoxygenation. Unsaturation adjacent to the epoxide linkage seems to be required, in order to form the radical-anion intermediate.

Desulfurization of sulfides

Equally instructive as to the role of ligands on chemical reactivity is the desulfurization of aryl sulfides by nickel(0) complexes (25). Dibenzothiophene can be cleaved by (bipy)(COD)$\mathrm{Ni}$ in THF to form nickel-containing precursors to bipheny1 (26), as shown in Fig. 12,

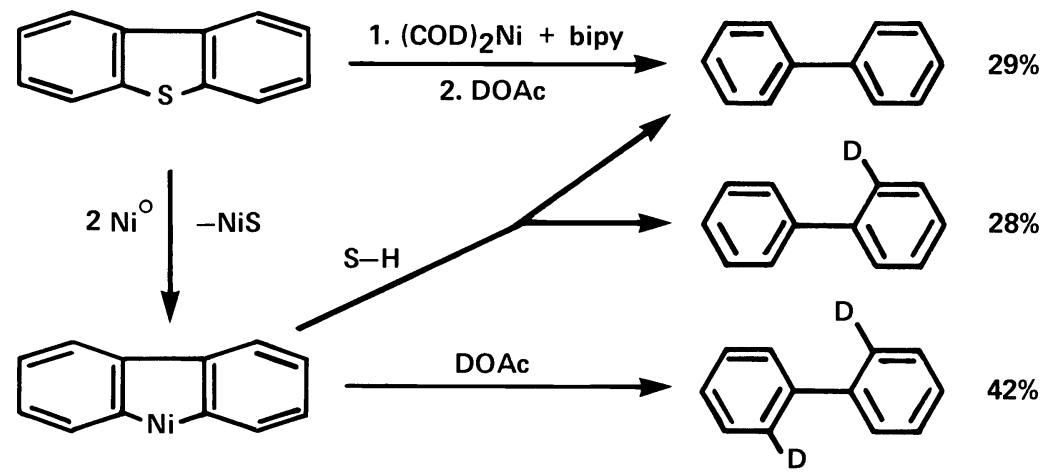

Fig. 12 Desulfurization of dibenzothiophene by nickel(0) complexes

whereas heterocycles such as phenoxathiin, (Fig. 13), and phenothiazine, can be desulfurized with the formation of dibenzofuran and carbazole, respectively.

The nature of the ligand is crucial to the desulfurization: whereas (COD) ${ }_{2} \mathrm{Ni}$ gives no significant cleavage of dibenzothiophene, various amines enhance the nickel atom's reactivity (Table 1). In agreement with previous comments, the specific ligands on the nickel atom have the greater influence on reactivity but the solvent's character can also play a role. Thus with (bipy)(COD)Ni, THF fosters desulfurization over that observed with benzene or ether. 


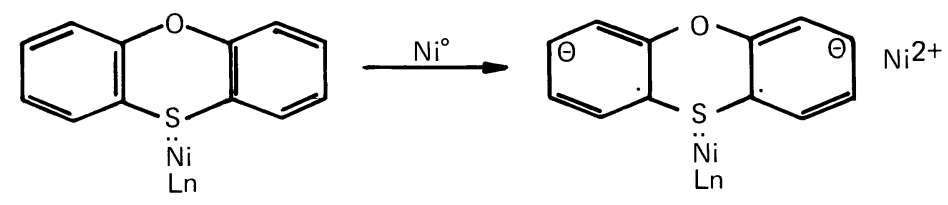

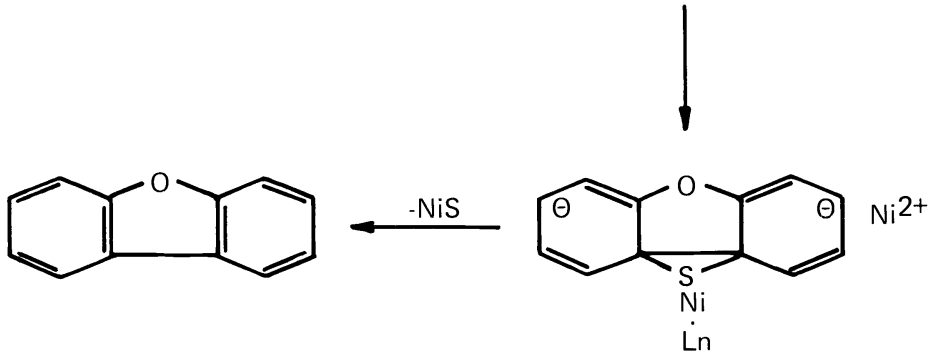

Fig. 13 Sulfur extrusion from phenoxathiin by nickel(0) complexes

TABLE 1. Effect of ligands on the reactivity of nickel(0) complexes

Ligand

Bipyridyl

4-Dimethylaminopyridine

1,10-Phenanthroline

Pyridine

HMPT

TMEDA

1,8-Bis(dimethylamino)naphthalene

\section{Biphenyl (\%)}

45

21

16

14

14

8

7
Conditions : $55^{\circ} \mathrm{C}$ in THF with 2 equiv. $(\mathrm{COD})_{2} \mathrm{Ni}$; HOAc work-up.

The nickel(0) complexes themselves, as well as an analogous cobalt complex, (COD) ( $\left.\mathrm{C}_{8} \mathrm{H}_{13}\right) \mathrm{Co}_{\text {, }}$ (27), show an enhanced desulfurizing activity when co-reacted with $\mathrm{LiAlH}_{4}$. Essentially quantitative yields of biphenyl are obtained from dibenzothiophene.

Toward substituted dibenzothiophenes, the reactivity of the (bipy)(COD)Ni reagent accords well with the occurrence of an SET process (Fig. 14):<smiles>[R20]c1cc2sc3cc([R20])c([R7])cc3c2cc1[R20]</smiles>

$\mathbf{R}_{1} \quad \mathbf{R}_{2}$

H H

Me H

$\mathrm{H}$
H<smiles>c1ccc(-c2ccccc2)cc1</smiles>

Yield (\%)
12

$<5$

Fig. 14 Reactivity of dibenzothiophenes toward nickel(0) complexes The lessening reactivity in the sequence, $\mathrm{DBT}>2,8-\mathrm{Me}_{2} \mathrm{DBT}>3,7-\mathrm{Me}_{2} \mathrm{DBT}$, parallels the increasingly negative values of the reduction potentials: $-2.432 ;-2.452 ;-2.563 \mathrm{~V}$. Furthermore, ESR measurements of the dibenzothiophene radical-anion show the greatest electron spin at $\mathrm{C}_{3}$ and $\mathrm{C}_{7}$, with the less at $\mathrm{C}_{2}$ and $\mathrm{C}_{8}$. Placement of electron-donating groups (Me) at these sites in dibenzothiophene should destabilize the radical-anion intermediate in the order: $\mathrm{DBT}>2,8-\mathrm{Me}_{2} \mathrm{DBT}>3,7-\mathrm{Me}_{2} \mathrm{DBT}$ (Fig. 15). Thus, both ESR and reduction potential measurements show that the radical-anions should decrease in stability in the same order as the observed reactivity. The greater energy necessary to add an electron to the dibenzothiophene system is reflected in a lesser reactivity in desulfurization (26). 
Me<smiles>Cc1ccc2sc3ccc(C)cc3c2c1</smiles><smiles>Cc1ccc2c(c1)sc1cc(C)ccc12</smiles>

Fig. 15 Contributory resonance structures for dibenzothiophenes

Oligomerization of alkynes

The cyclotrimerization of alkynes by nickel(0) is proposed to ensue through a sequence of nickel heterocycles, as depicted in Fig. 16 $(28,29)$. Evidence for the nickelacyclopropene<smiles>Cc1ccccc1</smiles>

$\mathrm{PhC} \equiv \mathrm{CPh}$

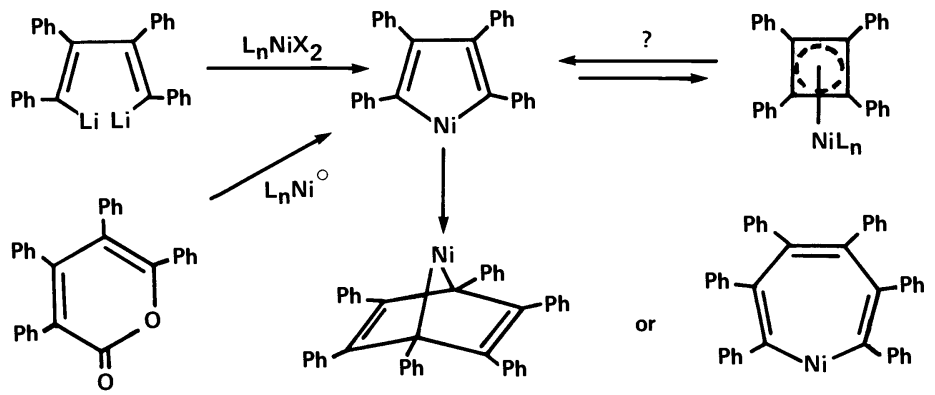

Fig. 16 Proposed intermediates and interconversions for the oligomerization of alkynes by nickel(0) complexes

and nickelacyclopentadiene intermediates rests both on isolation $(28,29)$ and on chemical trapping (Figs. 17,18) $(28,30)$. However, it is not yet known whether a nickelacycloheptatriene or its bicyclic isomer is involved in the final stage of trimerization. On the other hand, there is evidence that cyclopropenones, $\alpha$-pyrones and even cyclobutadienenickel(0) complexes can form nickel intermediates involved in the cyclotrimerization of alkynes $(28,30)$.

The role of ligands in forming and stabilizing these nickel intermediates is pronounced. Thus, the $1: 1$ pi-complex, which is depicted in Figs. 16 and 17 as a nickelacyclopropene, is stabilized markedly by bipyridyl and can be treated with $\mathrm{Me}_{3} \mathrm{SiN}=\mathrm{C}$ to yield carbonylation and hydrocyanation products (>80\%). In the absence of bipyridyl, the formation of the $2: 1$ alkyne-nickel adduct, nickelacyclopentadiene, is favored and it can be trapped with $\mathrm{Me}_{3} \mathrm{SiN}=\mathrm{C}$ to give a good yield of tetracyclone (Fig. 18). The overall cyclotrimerization of alkynes represents a sequence of oxidative addition, insertions and reductive elimination.

Although it is not yet clear whether cyclobutadiene-nickel(0) intermediates are formed from alkynes in the course of such cyclotrimerizations, it is known that reduction of tetraphenylcyclobutadiene-nickel dibromide by $t-B u L i$ in the presence of $\mathrm{Ph}_{3} \mathrm{P}$ does give an active alkyne-trimerization catalyst (31). Under these conditions, the cyclobutadiene ring may be cleaved to yield a nickelole. That certain four-membered rings can be cleaved by nickel(0) to form nickeloles has been established by the behavior of biphenylene toward $\left(\mathrm{Et}_{3} \mathrm{P}\right){ }_{4} \mathrm{Ni}$ in THF solution at low temperatures. 


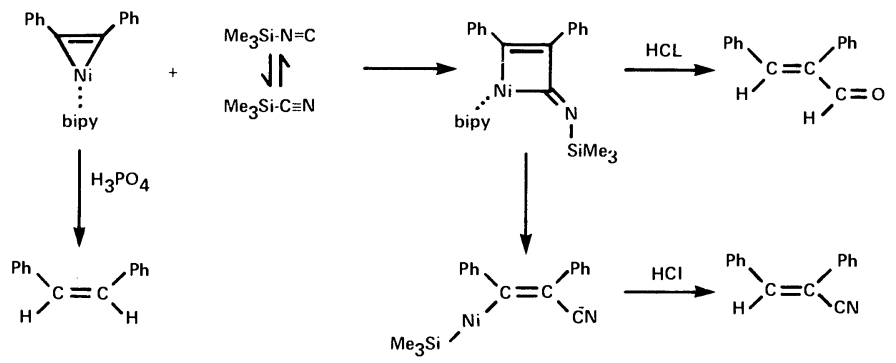

Fig. 17 Trapping of the $1: 1$ alkyne-nickel(0) complex by $\mathrm{Me}_{3} \mathrm{SiN}=\mathrm{C}$

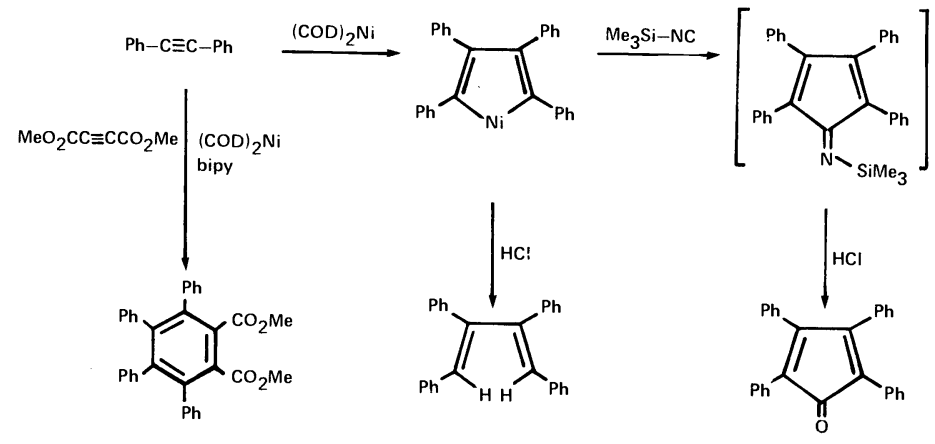

Fig. 18 Trapping of the $2: 1$ alkyne-nicke1(0) complex by $\mathrm{Me}_{3} \mathrm{SiN}=\mathrm{C}$ The golden brown nickelole depicted in Figs. 19 and 20 (or possibly its dimer) is rapidly formed in high yield. Its response to $\mathrm{CO}$, DOAc and tolane is exactly that expected for a dibenzonickelole:
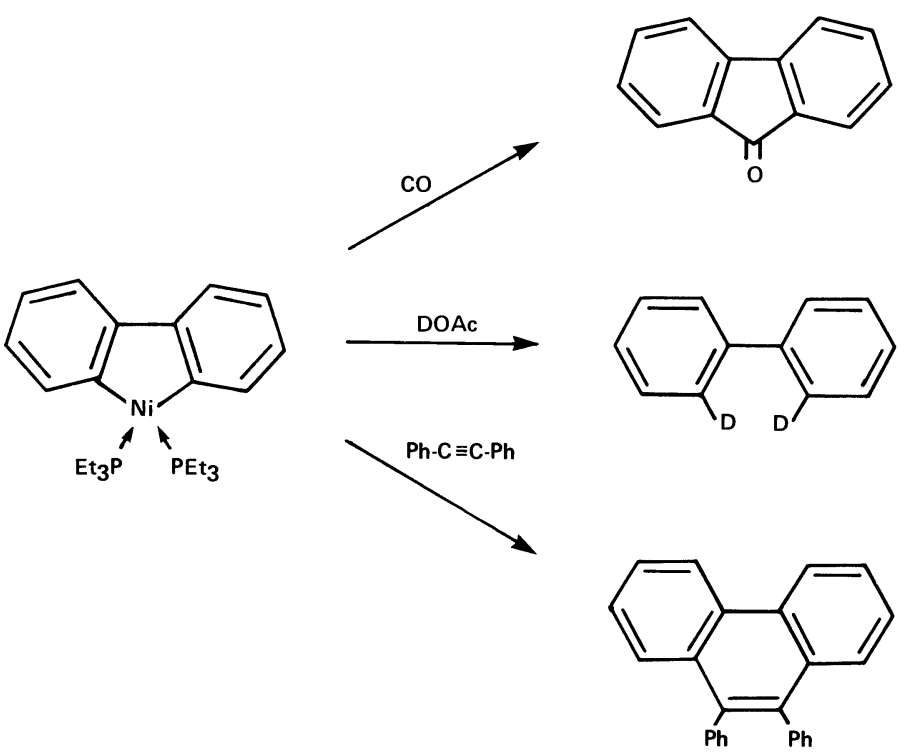

Fig. 19 Reactions of the dibenzonickelole system

Upon standing, the brown nickel complex loses one $\mathrm{Et}_{3} \mathrm{P}$ and rearranges to the green dimer shown in Fig. 20. The crystal structure of this unusual nickel heterocycle has been determined by Drs. Krueger and Tsay at the Max Planck Institut fuer Kohlenforschung. In addition to a nickel-nickel bond, each nickel is sigma-bonded to one ring and pi-bonded to to the other. Carbonylation yields a mixture of tetraphenylene and the cyclononatetraenone; thermolysis leads to tetraphenylene (32). In the behavior of this nickelole derived from biphenylene, it is possible that we have uncovered not only a model intermediate for cyclotrimerization of alkynes, but also a model for the Reppe cyclotetramerization of acetylene to cyclooctatetraene. One could imagine that both processes may involve the stepwise formation of nickelacyclopentadienes or nickeloles. Whether the resulting nickelole reacts with more alkyne to yield benzene derivatives or undergoes autodimerization, like the dibenzonickelole, to yield cyclooctatetraene derivatives may depend upon the stability of the 
<smiles>c1ccc2c(c1)-c1ccccc1-2</smiles>

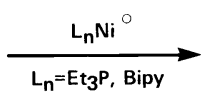<smiles>CCCCn1c2ccccc2c2ccccc21</smiles>
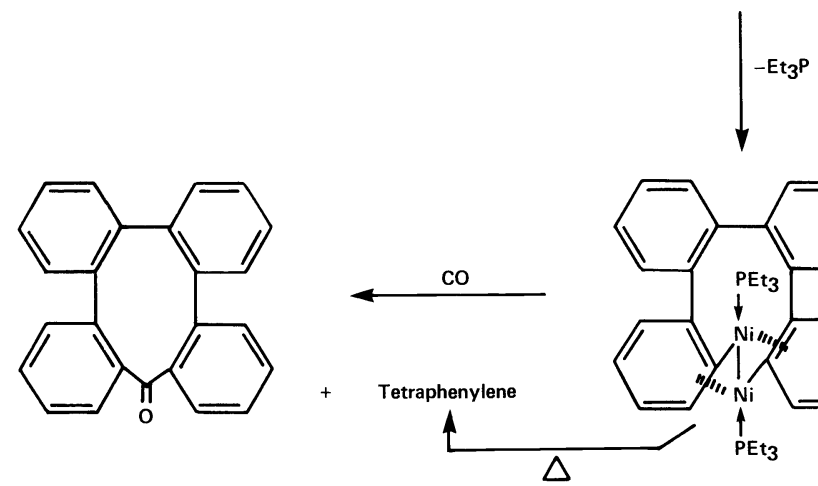

Fig. 20 Formation and rearrangement of the dibenzonickelole system intermediates and the reactivity of the specific alkyne. The implications of this suggestion are receiving our earnest experimental efforts.

\section{ELECTRON TRANSFER WITH TRANSITION METAL ALKYLS}

Because of the accessibility of multiple oxidation states, transition metal alkyls would also be expected to undergo SET processes with facility. The pervading tendency of such alkyls to undergo homolysis of their carbon-metal bonds is an obvious manifestation of SET reactions. A more unusual illustration of such processes is the occurrence of electron transfer subsequent to a typical carbometallation reaction, as exemplified in Figs. 21 and 22 . In the former figure, allyl(bis-cyclopentadieny1)titanium (III) reacts rapidly with benzophenone, but the resulting adduct must then undergo SET to yield the allyl(dipheny1)methyl radical, which then dimerizes (33).

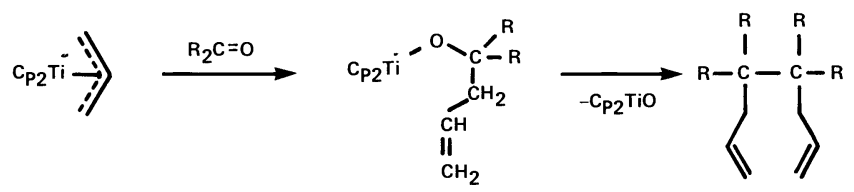

Fig. 21 Reaction of allyl(bis-cyclopentadienyl)titanium with $\mathrm{R}_{2} \mathrm{C}=0$ In the latter figure, some SET reactions of the bis-cyclopentadienyltitanium (IV)methylenezinc halide complex are shown. The coupling of arylmethyl halides is clearly an SET process, as is the dimerization of certain titanacyclobutenes formed from alkynes. Rupture of the titanium(IV)-allylic ring bond produces titanium(III)-substituted allylic radicals, which then couple to yield 1,5-hexadienes (34).

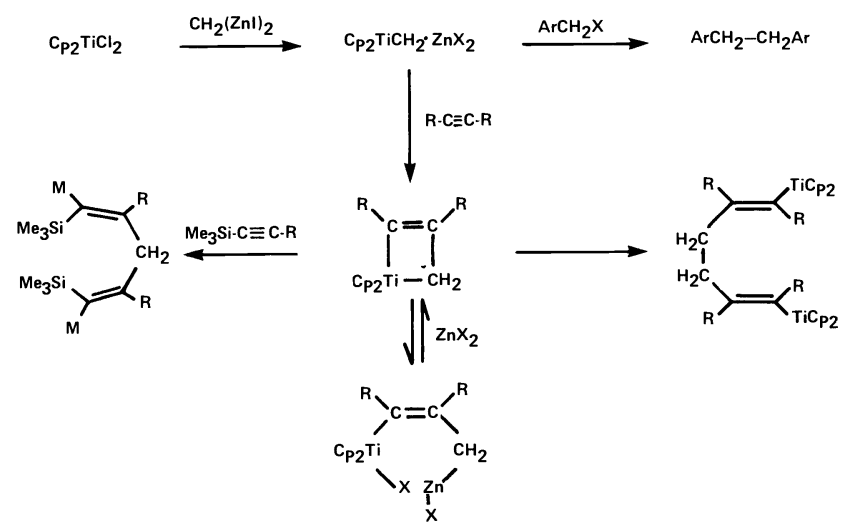

Fig. 22 Reactions of the titanocene-methylene.znx 2 complex 


\section{CONCLUS ION}

For both main-group and transition metals, useful parallels in behavior can be drawn between zerovalent metals and their organometallic compounds. Rundle's fruitful analogy ascribes to both metals and organometallics a certain degree of electron delocalization, which makes both systems potential sources of electrons. By experiment, one can observe SET processes with either zerovalent metals or organometallics. Furthermore, by invoking Born-Haber cycles and Hammond's postulate, one can rationalize the observed effects of metal structure, solvent, ligand and substrate on the ease of electron transfer.

\section{ACKNOWLEDGMENTS}

The author is grateful to the National Science Foundation and the Department of Energy for the support of the research reported here.

\section{REFERENCES}

1. G.E. Coates, M.L.H. Green and K. Wade, Organometallic Compounds, 2 vols., Methuen, London (1967-1968).

2. J.J. Eisch, The Chemistry of Organometallic Compounds, pp. 13-23, Macmillan, New York (1967).

3. J.J. Eisch, Organometallic Syntheses, Vo1. 2, pp. 38-48, Academic Press, New York (1981).

4. Reference 2, pp. 80-94.

5. F.F. B1icke and L.D. Powers, J. Am. Chem. Soc. 51, 3378 (1929).

6. E.C. Ashby, Pure and Applied Chemistry 52, 545 (1980).

7. R.E. Rundle, J. Phys. Chem. 61, 45 (1957).

8. J.E. Mulvaney, Z.G. Gardlund and S.L. Gardlund, J. Am. Chem. Soc. 85, 3897 (1963).

9. G. Wilke and H. Mueller, Justus Liebigs Ann. Chem. 629, $222(1960)$.

10. A.N. Nesmeyanov, A.E. Borisov and I.S. Saveleva, Izv. Aka. Nauk SSSR Ser. Khim. 1034 (1959).

11. G.S. Hammond, J. Am. Chem. Soc. 77, 334 (1955).

12. W. Schlenk and R. Ochs, Ber. Dtsch Chem. Ges. 49, 608 (1916).

13. E.J. Panek, J. Am. Chem. Soc. 95, 8460 (1973).

14. J.J. Eisch, D.D. Kaska and C.J. Peterson, J. Org. Chem. 31, 453 (1966).

15. J.J. Eisch, J. Org. Chem. 28, 707 (1963).

16. J.J. Eisch and R.J. Beuhler, J. Org. Chem. 28, 2876 (1963).

17. J.J. Eisch and G. Gupta, J. Organomet. Chem - 50, C23 (1973).

18. J.J. Eisch and G. Gupta, J. Organomet. Chem. 168, 139 (1979).

19. J.J. Eisch and L.E. Smith, J. Organomet. Chem. 50, C18 (1973).

20. J.J. Eisch and J.E. Galle, unpublished studies, 1980.

21. R.L. Smorada and W.E. Truce, J. Org. Chem. 44, 3444 (1979).

22. J.J. Eisch and M. Behrooz, unpublished studies, 1983.

23. J.J. Eisch and J.E. Galle, J. Org. Chem. 44, 3277 (1979).

24. J.J. Eisch and K.R. Im, J. Organomet. Chem. 139, C45 (1977).

25. J.J. Eisch and K.R. Im, J. Organomet. Chem. 139, C51 (1977).

26. J.J. Eisch, L.E. Hallenbeck and K.I. Han, J. Org. Chem. 48, 2963 (1983).

27. J.J. Eisch and A. Piotrowski, unpublished studies, 1982 .

28. J.J. Eisch and J.E. Ga11e, J. Organomet. Chem. 96, C23 (1975).

29. H. Hoberg and W. Richter, J. Organomet. Chem. 1955,355 (1980).

30. J.J. Eisch, A.A. Aradi and K.I. Han, Tetrahedron Lett. 24, 2073 (1983).

31. J.J. Eisch and A.A. Aradi, unpublished studies, 1982 .

32. J.J. Eisch, A.A. Aradi and A. Piotrowski, Third International Symposium on Homogeneous Catalysis, Milan, Italy, Aug. 31, 1982.

33. J.J. Eisch and M. Bolesławski, unpublished studies, 1983.

34. J.J. Eisch and A. Piotrowski, Tetrahedron Lett. 24, 2043 (1983). 